\title{
Die Bedeutung der Hyperämie bei der Behandlung von paretischen Erscheinungen neuritischer Herkunft.
}

\author{
Von \\ Prof. Michael Lapinsky (Kiew). \\ (Eingegangen am 28. Juli 1913.)
}

Im Laufe der letzten 10 Jahre hatte ich Gelegenheit, mehr als 15 Fälle von schwerer chronischer Polyneuritis zu beobachten, die sich entweder auf dem Boden einer Arsenikvergiftung (3 Fälle), oder als Folge von Alkoholmißbrauch (2 Fälle), oder als Begleiterscheinung der Diphtherie, der Angina follicularis, des Typhus und anderer Infektionskrankheiten entwickelt hatte. Viele von diesen Kranken hatte ich Gelegenheit nach 2, 3 bis 4 Jahren seit dem Beginn der Erkrankung wiederzusehen, nach dem sie sich, allerdings aber ohne jeden sichtbaren Erfolg, allen möglichen starkwirkenden Behandlungsmethoden unterworfen hatten. Einige dieser Kranken behandelte ich selbst ununterbrochen im Laufe von 3-4 Jahren und brachte bei ihnen das gesamte für derartige Fälle empfohlene therapeutische Inventar zur Anwendung, jedoch ebenfalls ohne großen Erfolg. In dieser Hinsicht stehen meine Beobachtungen in vollkommenem Einklang mit den Erfahrungen anderer Ärzte, deren diesen Gegenstand betreffende Meinung sich gegenwärtig im allgemeinen dahin zusammenfassen läßt, daß die Behandlung von Kranken mit chronischen Neuritiden, wenn schon $1 / 2$ Jahr seit dem Beginn der Erkrankung vergangen, eine im höchsten Grade undankbare Aufgabe darstellt: die Patienten werden jahrelang elektrisiert, nehmen Bäder, werden massiert, treiben Gymnastik usw., doch weder sie selbst, noch die behandelnden Ärzte werden dadurch in irgendeiner Weise befriedigt. Das ist der Grund, weshalb die praktischen Ärzte die Prognose bei in das chronische Stadium übergegangenen Neuritiden nur mit äußerster Vorsicht stellen.

Angesichts derartiger, wenig tröstlicher Resultate sei es mir gestattet, hier einige Krankengeschichten, in denen die Neuritiden chronisch verliefen, wo jedoch nichtsdestoweniger die angewandte Behandlung durchaus zufriedenstellende Resultate ergeben hat, mitzuteilen.

I.

1. L. M. Ch. $12 \mathrm{Jahr}$ alt, trat am 5. September 1909 mit Klagen über Schmerzen und Schwäche im linken Bein als ambulatorische Kranke in meine Behandlung ein. 
Linker Fuß und Unterschenkel blaß und eyanotisch, fühien sich kalt an. Puls in den aa. pedis dorsalis und tibialis postica nur mit Mühe palpabel; rechterseits ist er in diesen Arterien normal. Kein Ödem; Haut atrophisch. Der linke Fuß ist im allgemeinen dünner als der rechte; der Umfang des linken Unterschenkels unterhalb der Tuberositas tibialis um $3 \mathrm{~cm}$ kleiner als rechterseits; der FuB im Chopartschen Gelenk um $1 \mathrm{~cm}$ dünner als der rechte; der Umfang des linken Oberschenkels in der Mitte und im unteren Drittel um $2 \mathrm{~cm}$ dünner als der des rechten. Dàs ganze rechte Bein vollkommen normal. Linker Fuß in der Stellung eines Pes varo-equinus. Die Zehen nach unten gebogen. Passive Bewegungen in den Gelenken des rechten Beines völlig frei, während dieselben in den Zehengelenken und im Sprunggelenk des linken allerdings ihre volle Amplitude zu entwickeln vermögen, aber doch in Abhängigkeit von den Muskeln, sowohl in der Extension, als auch in der Flexion etwas erschwert sind; die passiven Bewegungen im Knie sind frei. Die aktiven Bewegungen im rechten Bein vollkommen frei, im linken - nur im Kniegelenk; im Sprunggelenk sind sie nur in der Richtung der Plantarflexion möglich; doch auch diese letzteren sind im Vergleich zum rechten Fuße äußerst schwach; die willkürliche Hebung des Fußes ist vollkommen unmöglich; aktive Bewegungen der Zehen sind nur in der Flexionsrichtung angedeutet, sehr schwach und von nicht voller Amplitude; eine aktive Extension der Zehen wird überhaupt nicht erhalten. Empfindlichkeit gegen Nadelstiche im linken Fuß und im Unterschenkel auf der äußeren, hinteren und vorderen Oberfläche bis zum Niveau des Knies nicht vorhanden; normal ist sie auf der inneren Seite dieser Körperteile, am Knie und am Oberschenkel, d. h. im Bereich des N. saphenus int.; im rechten Bein ist die Sensibilität normal. Der Sohlenreflex fehlt auf der linken Seite und ist auf der rechten normal; der Achillessehnenreflex fehlt am linken FuB und ist rechtsseits normal; der Patellarsehnenreflex ist linkerseits träge, rechterseits normal. Die Untersuchung mit dem galvanischen und faradischen Strom ergab folgendes:

Cialvanischer Strom.

Links

N. tibialis 7,0 M.-A. Sehr träge Zuckung - KZS $<$ AZS

N. peroneus 9,0 M..A. Sehr träge Zuckung, - KZS $<$ AZS

\section{Links}

Faradischer Strom.

N. tibialis Bei der Annäherung der Rollen des Schlittenapparats bis auf $5 \mathrm{~cm}$ keinerlei Zuckung im M. triceps surae. Das weitere Aneinanderrücken der Rollen sehr schmerzhaft.

N. peroneus Bei $4 \mathrm{~cm}$ Rollenabstand träge Zuckung.
Rechts

3,0 M.-A., momentane Zuckung $-\mathrm{KZS}>\mathrm{AZ}$ 1,5 M.-A. momentane Zuckung.

Rechts

Bei einem Rollenabstand von $10 \mathrm{~cm}$ normale Zuk. kung im M. soleus.

Bei $11 \mathrm{~cm}$ Rollenabstand normale Zuckung.

Die anamnestischen Angaben sind in diesem Falle äußerst unklar: die Mutter vermochte nicht mit Sicherheit den Beginn der Erkrankung ihrer Tochter anzugeben, behauptete aber, daß die Schwäche im Fuße besonders stark vor 2 Jahren bemerkbar war, als sie gefallen und ihr linkes Knie verletzt hatte. Es gelang festzustellen, daß die Kranke vor mehreren Jahren die ansteckenden Kinderkrankheiten durchgemacht, sowie an Mandelentzündung gelitten hatte u. dgl. m.

$\mathrm{Da}$ diese Anamnese keinerlei Hinweise darauf enthielt, daß der Fall eine Erkrankung des Kniegelenks mit Einklemmung von Zweigen des N. ischiadicus, 
oder eine ständige Verunstaltung, oder eine zeitweilige Schwellung des Knies, oder des ganzen Beines nach sich gezogen, oder einen Hautriß mit nachfolgender Temperaturerhöhung usw. hervorgerufen hatte, so läßt sich diesem Trauma im gegebenen Falle überhaupt keine besondere Bedeutung beimessen, und man ist gezwungen, die ganze Erkrankung als eine Neuritis der NN. tibialis und peroneus aufzufassen, die sich vor recht langer Zeit, vielleicht im Verlaufe der einen oder anderen ansteckenden Kinderkrankheit herausgebildet. Zugunsten einer solchen Diagnose sprach das gesamte klinische Bild, und zwar die Atrophie der Wadenmuskulatur, das Betroffensein der motorischen Sphäre und der Sensibilität nur in den Grenzen des Fußes und Unterschenkels, vorzugsweise im Bereich der NN. tibialis und peroneus, das Fehlen der Hautreflexe und des O p penheim schen Reflexes, sowie des Achillessehnenreflexes; vielleicht war bis zu einem gewissen Grade auch der linke N. cruralis an der Erkrankung beteiligt, zugunsten welcher Annahme der träge linke Patellarreflex sprach. Gänzlich auszuschließen war der Gedanke an irgendeine Myopathie oder eine chronische oder akute Poliomyelitis angesichts der scharf ausgeprägten Herabsetzung der Sensibilität der Vorder-, Außen- und Hinterfläche des Unterschenkels, und zwar im Bereiche der NN. cutaneus surae ext. und peroneus superficialis. In Anbetracht des Vorhandenseins der Degenerationsreaktion einerseits und der Herabsetzung der Sensibilität innerhalb der genauen anatomischen Grenzen der peripheren Nerven der betreffenden Extremität anderseits war auch Hysterie völlig auszuschließen.

Es wurde folgende Behandlung angeordnet: 1. 10 Minuten lang währende hydraulische Massage des linken Fußes und Unterschenkels in einem heißen Wasserstrom von $35-40^{\circ} \mathrm{R}$ bei einem Druck von $1 / 2-3 / 4 \mathrm{Atm}$., so daß $\mathrm{B}$ der mechanische Massageeffekt sowohl mit der Hand, als auch durch den Wasserdruck erreicht wurde. Nach 10 Minuten währender Bearbeitung der kranken Extremität in der angegebenen Weise nahm deren Haut eine hochrote Färbung an und fühlte sich nun nicht mehr kalt, sondern warm an; 2. hierauf wurde das Bein 10 Minuten lang der Einwirkung eines Strahles von strömenden Wasserdampf ausgesetzt, worauf diese Hyperämie sich noch weiter entwickelte; 3 . sodann sab die Patientin 10 Minuten lang in einem Bade von $29^{\circ} \mathrm{R}$., in das die Elektroden eines dreiphasischen sinusoidalen Stromes in der Weise getaucht wurden, daß sich die eine Elektrode zwischen den Schulterblättern, die zweite auf dem Bauche befand, während die Patientin ihre Fußsohle auf die dritte stützte; 4. zum Schlusse erbielt die Kranke eine kurzdauernde temperierte Dusche.

Bereits am 5. Tage seit dem Beginn dieser Behandlung änderten sich die Hautfarbe der betroffenen Extremität und die Temperatur der letzteren in scharf ausgeprägter Weise. Die Cyanose verschwand während der hydropathischen Prozeduren und trat erst gegen Abend in nur sehr schwachem Grade wieder auf. Die Haut des kranken Fußes fühlte sich wärmer an, und den ganzen Tag über hatte die Kranke nun nicht mehr das unangenehme Kältegefühl in demselben. Nach Verlauf eines Monats seit dem Beginn der Behandlung begann der Fuß, der bis dahin in der Stellung eines Pes varo-equinus verharrt hatte, eine der Norm nahekommende Stellung anzunehmen, und der von ihm und dem Unterschenkel gebildete Winkel näherte sich $90^{\circ}$. Nach 2 Monaten wurde die Hautfarbe des Fußes und Unterschenkels normal; die Haut fühlte sich warm an, der Puls in den AA. pedis. dorsalis und tibialis postica erwies sich bei der Palpation als normal; die Zehen des linken Fußes und der Fuß selbst waren nach allen Richtungen vollkommen frei beweglich, wenn auch viel schwächer als auf der rechten Seite; die Sensibilität wurde normal; der Kniereflex gleichfalis; der Achillessehnenreflex war allerdings sehr träge, aber zweifellos deutlich ausgeprägt; die galvanische Erregbarkeit der NN. tibialis und peroneus, KZs $>A N Z$, blieb linkerseits noch schwächer 
als auf der rechten Seite, weshalb man genötigt war, für die Nervenstämme dieser Seite einen um 2-3 M.-A. stärkeren galvanischen Strom einzuführen als auf der entgegengesetzten; der faradische Strom ergab eine deutliche, wenn auch träge Zuckung von den linken NN. tibialis und peroneus bei einem Rollenabstand von $7 \mathrm{~cm}$ für den $\mathrm{N}$. tibialis und von $9 \mathrm{~cm}$ für den N. peroneus.

2. Frl. Sch. zeichnete sich durch eine gute Gesundheit und große Widerstandsfähigkeit aus. Vor 3 Jahren war sie in einen politischen Prozeß verwickelt worden und erkrankte, als sie sich im Zusammenhang hiermit in Cntersuchungshaft im Gefängnis befand, an Gefängnisgangrän der Lunge. Angesichts dieser schweren Erkrankung, die sie ins Grab zu bringen drohte, wurde sie aus der Haft entlassen und fuhr aufs Land, wo sehr bald šchmerzen im ganzen Körper, Abmagerung und Schwäche, insbesonders der Hände und Füße bis zu völliger Immobilität derselben und trophische Störungen an den Füßen in Gestalt von eiterigen Geschwüren auftraten, die besonders in den distalen Fingerphalangen lokalisiert waren. Die Nägel der drei äußeren Zehen des rechten Fußes wurden dunkel. Angesichts solcher drohender Symptome wurde die Patientin nach Kiew gebracht und im Oktober des Jahres 1907 in das Krankenhaus des Pokrowski-Klosters (Abteilung des Herrn Dr. W. P. Demtschenko) untergebracht. Hier wurde bei der Besichtigung der Kranken durch viele Ärzte und auch durch mich folgendes gefunden: Sehr erschöpftes Aussehen. Haut von ikterisch-cyanotischer Färbung. Die Füße und Hände cyanotisch verfärbt, schweiBig, fühlen sich kalt an. Die Schleimhäute cyanotisch. Die Nägel am linken Fuße sind von kleinen Geschwüren umgeben und zeigen Neigung zur Ablösung. Das subcutane Fettgerwebe ist stark geschwunden und läßt sich überall, insbesondere am Rumpf, auf der Brust und an den Oberschenkeln in Falten abheben. Die Gelenke sind frei und passiv beweglich, die Muskelmassen in ihrem Umfang verringert; besonders scharf ausge. prägt ist die Muskelatrophie an der Hand (am Thenar und Hypothenar); die grobe Muskelkraft der Hände ist so herabgesetzt, daß die Kranke nur mit Mühe einen Löffel in der Hand halten kann; alle Bewegungen im Schulter-, Ellenbogenund Radiokarpalgelenk sind jedoch möglich, wenn auch überaus verlangsamt; vollkommen fehlen die willkürlichen Bewegungen in den Zehen beider Füße and im Sprunggelenk; abgeschwächt sind sie in den Kniegelenken; bei Gehversuchen kann die Kranke nur mit großer Anstrengung die Beine über den Fußboden schleppen, ohne dazu instande zu sein, die Fußspitzen und Füße vom Boden zu erheben. Die Sensibilität ist in den distalen Teilen der Extremitäten herabgesetzt; weder Haut- noch Sehnenreflexe vorhanden. Degenerationsreaktion. Puls in den AA. pedis dorsales und tibiales posticae nur mit Mühe fühlbar. Systolisches Geräusch an der Herzspitze; scharf ausgeprägte Arhythmie.

Die Behandlung bestand in lokaler Elektrisation und Bädern.

Im April des Jahres 1908 wurde die Patientin behufs einer gynäkologischen Operation in die Heilanstalt von S. A. Gutstein übergeführt, wo auf einem Konsilium die Herren Prof. K. Wagner und Th. Ja nowski gleichfalls Herzerweiterung und Geräusche an der Spitze und Basis des Herzens feststellten. Der Zustand des Nervensystems lie $\mathbb{B}$ keinerlei Besserung erkennen.

Nach dem Verlassen der Heilanstalt nahm die Patientin 9 Monate lang galvanische warme Bäder von 28-29 $\mathrm{R}$. Außerdem gelangten Massage beider Hände und Füße und Faradisation der geschwächten Muskeln zur Anwendung.

Im November des Jahres 1908 besuchte mich die Kranke während meines ambulatorischen Krankenempfangs. Von beiden Seiten gestützt, konnte sie, in. dem sie die ganze Zeit über die unter der Last des eigenen Körpers zusammenbrechenden Beine nur mit Wühe nachzog, da es ihr an Kraft fehlte die Fußspitzen oder die Füße zu heben, langsam nur einige Schritte machen, um durch mein 
Sprechzimmer zu gehen. Bei der objektiven Untersuchung fand ich im Vergleich damit, was im Dezember des Jahres 1907 im Krankenhause des Pokrowski-Klosters festgestellt worden, fast keinerlei Veränderungen vor; übrigens konnte man von einer Verschlimmerung des Zustandes des Herzens reden; der Puls betrug 130-140, die linke Herzgrenze ging um 1 Fingerbreit über die linke Mammillarlinie hinaus, rechterseits wurde die Dämpfung durch die Mitte des Sternums gekennzeichnet; systolische und diastolische Geräusche wurden an allen Klappen auskultiert; Hände, Füße und Unterschenkel waren ödematös und cyanotisch; der Puls war in beiden Füßen nur mit Mühe palpabel. Willkürliche Bewegungen der Zehen und Sprunggelenke nicht möglich. Füße in Stellung des Pes varo-equinus. Es besteht Muskelatrophie, die am Thenar und Hypothenar, im Schultergürtel und an den Oberschenkeln besonders scharf ausgeprägt ist; infolge des starken Ödems der Füße und Unterschenkel war es schwer, den Zustand der Muskeln der Waden und der Dorsa pedum festzustellen. Taktile Sensibilität, Wärme- und Kälteempfindung waren an den Enden der Zehen nicht vorhanden, klärten sich aber in der Richtung nach oben allmählich, so daß sie im Oberschenkel und Rumpf normal waren; die gleichen Arten der Sensibilität waren in den Fingern nur leicht herabgesetzt. Das Schmerzgefühl, das in den distalen Teilen der Hände und Füße herabgesetzt war, erreichte an den Oberschenkeln und am Bauch allmählich wieder die Norm. Haut- und Sehnenreflexe an den Beinen gar nicht vorhanden; Sehnenreflexe an den Händen geschwächt. Nervenstämme sowohl an den Füßen, als auch an den Händen scharf ausgeprägt schmerzempfindlich auf Druck. Galvanische Reaktion in den NN. tibialis, peronei und crurales stark herabgesetzt (bis auf 10-12 M.A.), wobei AZS > KZS. Der auf die NA. tibiales und peronei applizierte faradische Strom rief sogar beim Aneinanderrücken der Rollen keinerlei Zuckungen hervor. An den Händen waren die gleichen Arten von elektrischer Erregbarkeit mäßig herabgesetzt. Hierauf trat die Kranke in eine private Heilanstalt ein, wo sie laut ihrer Aussage $1^{1 / 2} / 2$ Monate lang elektrische Bäder (offenbar mit dem galvanischen Strom) und unter hohem Druck stehende Duschen nahm.

14 Monate nach dieser Besichtigung, und zwar im Februar 1910, d. h. mehr als nach 21/2 Jahren seit dem Beginn der Erkrankung an Neuritis suchte sie als ambulatorische Patientin meine Behandlung auf. Ihre Klagen, sowie die Ergeb. nisse der objektiven Untersuchung waren die gleichen, wie vor 14 Monaten: Herzklopfen, Abmagerung der Hände, Ödem und Cyanose der Beine; die Kranke konnte wie früher die Zehen nicht bewegen, die Füße weder heben noch senken; nur mit Mühe war sie imstande die Beine im Knie zu beugen oder zu strecken; sie konnte nur mit Mühe bei fixierten Beinen den Rumpf aufrecht halten; ihre Hände waren ebenfalls geschwächt, immerhin konnte sie dieselben zum Essen, Schreiben usw. benutzen; wegen allgemeiner Schwäche war sie größtenteils gezwungen hilflos im Bett zu liegen; außerdem wurde sie von beständigen nagenden Schmerzen in beiden Beinen geplagt; Blasen- und Dickdarmfunktion völlig normal; gehen konnte die Kranke ausschlieBlich mit fremder Unterstützung und auch dann nur kurze Zeit, wobei sie die Beine mit großer Mühe bewegte und nach 10-15 Schritten gezwungen war sich zu setzen; das Treppensteigen verursachte ihr noch größere Anstrengung und war nur unter Beihilfe von $2-3$ Personen möglich. Puls 120-130. Äußere Herzgrenze 1 Finger breit nach links von der Mammillarlinie; systolische Geräusche vorhanden, insbesondere an der Herzspitze. Farbe der Haut und Schleimhäute cyanotisch wie früher. Haut an den Füßen, Unterschenkeln und Händen kalt. Puls an den Füßen in den AA. dorsales pedis und tibiales anticae nur mit Mühe fühlbar. Ödeme der Füße und Unterschenkel. Grobe Muskelkraft, Atrophie, Reflexe, Sensibilität und elektrische 
Reaktionen wie vor 14 Monaten. Schläft nachts wegen der Schmerzen in den Extremitäten, besonders in den Beinen, schlecht.

Die Behandlung bestand in mit starker in die Tiefe gehender und kutaner Hyperämie einhergehenden Prozeduren, und zwar in 5-10 Minuten währender hydraulischer Massage der Füße, Unter- und Oberschenkel bei einer Temperatur von $29^{\circ}-10^{\circ} \mathrm{R}$, Dampfspray auf Beine und Arme, Heißluftbädern im geschlossenen Schwitzkasten bis zum Eintreten reichlicher Schweißabsonderung, in sinusoidalen Bädern, Duschen und in partiellen Heißluftlichtbädern der Füße und Unterschenkel. Hiervon waren nur die hydraulische Massage, der Dampfspray und die Heißluftbäder vollkommen neue Verordnungen für die Kranke; was nun die sinusoidalen Ströme (3 Pole) anbelangt, so mögen, obschon die Kranke offenbar bisher keine derartige Behandlung erfahren hatte, die ihr früher verordnete Galvanisation und Faradisation immerhin eine den sinusoidalen Bädern analoge Wirkung aufgewiesen haben. Was nun die obenerwähnten bei der Kranken bisher noch nicht versuchten trten der Thermotherapie anbelangt, so äußerte sich ihre Wirkung in einer starken Hyperämie der Haut, die sich tagsüber viele Stunden lang hielt. Nach 10 tägiger Behandlung in der oben geschilderten Weise begann die Kranke in ihren Füßen fast während des ganzen Tages, an dem die Heilprozeduren vorgenommen wurden, ein Gefühl von Wärme zu empfinden; die cyanotische Hautverfärbung der Füße und deren Abkühlung traten erst abends auf; der Puls in den AA. dorsales pedum und tibiales posticae wurde völlig normal. Die Kranke besuchte mich 4 Monate lang; die ersten 6 Wochen wurde sie unter fremder Beihilfe auf einer Droschke befördert, in der Mitte des 2. Monats kam sie selbständig auf einer Droschke angefahren und konnte später bereits die 300 Schritte bis zum Tramway zu Fuß zurücklegen; gegen Ende des 3. Monats legte sie den Hin- und Rückweg mit der elektrischen Bahn zurück, und das Treppensteigen machte ihr keine besonderen Schwierigkeiten mehr.

Die inzwischen erfolgten wiederholten Untersuchungen hatten folgende Veränderungen in ihrem Zustande erkennen lassen. Die Cyanose und das Kältegefühl in den distalen Körperteilen verschwanden gegen Ende der 3. Woche. In der 4 . Woche gingen die Ödeme zurück, und nur eine mäßige teigige Beschaffenheit bestand noch. In der 7. Woche der Behandlung wurden die Herzgrenzen normal, und die Herzgeräusche hörten auf. In der 8. Woche fiel der Puls auf 80-86 in 1 Minute; jetzt wurde derselbe sowohl an den Füßen, als auch an den Händen völlig normal. Die Sensibilität in allen ihren Formen war in der 9. Woche endgültig wiederhergestellt. In der Mitte des 3. Monats der Behandlung begann die Kranke willkürlich die Zehen beider Füße zu strecken und zu beugen. Und noch später, und zwar ungefähr gegen Ende des 3. Monats, begannen die ersten Anzeichen von willkürlichen Flexions- und Extensionsbewegungen in den Sprunggelenken sich einzustellen. In der Mitte des 4. Monats traten wenn auch nur sehr schwache Kniereflexe auf; die Achillessehnenreflexe fehlten; zu dieser Zeit wurden die Reflexe von den MM. tricipites und supinatores longi fast vollkommen normal. Die elektrische Reaktion wies vor dem Fortgang der Patientin aus der Behandlung im Juni 1910 immerhin noch eine beträchtliche Herabminderung gegenüber der Norm auf, doch die NN. tibiales und peronei reagierten bereits auf den faradischen Strom, und die galvanische Reaktion ergab AZS $<$ KZS. Die Patientin muBte eingetretener Umstände halber Kiew verlassen.

In diesem schweren Polyneuritisfalle wurde mit der Anwendung der Hyperämie erzeugenden Prozeduren nach Verlauf von mehr als $2^{1 / 2}$ Jahren seit dem Beginn der Erkrankung angefangen nach voraufgegangener lange Zeit hindurch fortgesetzter und erfolgloser Behand- 
lung mit verschiedenen Verfahren, insbesondere mit Elektrizität; den bedeutenden Erfolg, der im Laufe der 4 Monate währenden systematischen Anwendung der Thermotherapie erreicht wurde, hatte man ausschließlich auf Rechnung der Wirkung der Hyperämie erzeugenden Prozeduren zu setzen.

3. General D., 49 Jahr alt, wurde im Jahre 1906 während der Revolution in Sebastopol (Krim) durch eine explodierende Bombe an beiden Beinen verletzt; hierbei erhielt er am linken Bein außer tiefen Brandwunden auch noch eine Verletzung des $\mathrm{N}$. peroneus, weshalb er nach Wiederherstellung von den Brandwunden nicht imstande war, die Zehen des betroffenen Fußes zu bewegen und den letzteren im Sprunggelenk zu beugen. Die Sensibilität im Fußrücken und in den Zehen war herabgesetzt. Auf den Vorschlag des Herrn Dr. D. A. Abuladse kam der Kranke im März 1910 nach Kiew, wo er ambulatorisch der obenerwähnten Behandlung unterworfen wurde.

Der linke Fuß und der linke Unterschenkel erschienen bei seinem Eintritt im Vergleich zur entsprechenden rechten Extremität dünn und abgemagert; die Haut dieser Körperteile war kalt und blaß-cyanotisch. Der Puls in den linken AA. pedis dorsalis und tibialis postica kaum fühlbar, in den übrigen gut palpabel. Die Zehen nach unten gebogen; der Fuß hing ein wenig nach unten und außen. Die passive Beweglichkeit in den Zehengelenken, dem Sprung- und Kniegelenk vollkommen frei und schmerzlos. Die aktive Beweglichkeit war nur im Kniegelenk völlig regelrecht; in den Gelenken der Zehen war sie jedoch gar nicht vorhanden: der Kranke vermochte dieselben weder aufwärts zu bewegen, noch stärker zu flektieren; aktive Bewegungen im Sprunggelenk waren nur in unbedeutendem Maße, und zwar etwas aufwärts (M. tibialis anticus) und noch weniger abwärts möglich. Die Sensibilität war im ganzen Fuß und im unteren Drittel des Unterschenkels mit Ausnahme der Innenseite dieser Körperbereiche herabgesetzt. Ptantar- und Achillessehnenreflex nicht vorhanden. Die Erregbarkeit der NN. tibialis und peroneus sin. durch den faradischen und galvanisehen Strom erhalten geblieben, aber auf 9 und $10 \mathrm{M}$.-A. herabgesetzt; KZS = AZS.

Diagnose: Traumatische Neuritis der linken NN. tibialis und peroneus.

Die Behandlung bestand in Hyperämie erzeugenden lokalen Prozeduren und sinusoidalen Strömen. Auf den linken Fuß wurde die hydraulische Massage appliziert, sodann der Dampfspray im Laufe von 10 Minuten, ein Sinusoidalbad von $32^{\circ} \mathrm{R}$ und eine heiße $\left(40^{\circ} \mathrm{R}\right)$ Dusche. Bereits nach 3 Tagen besserte sich die Blutzirkulation in den Füßen, und der Puls wurde sehr voll. Nach 4 Wochen begann D. aktive wenn anch nur sehr geringfügige Flexions- und Extensionsbewegungen der Zehen auszuführen. Leider war der Kranke durch seine Abreise aus Kiew gezwungen die Behandlung aufzugeben.

4. Frl. L...ch, Gouvernante von 40 Jahren, kam im Dezember 1912 wegen einer inveterierten Paralyse der linken Gesichtshälfte, die sie schon während dreier Jahre kurierte, in meine Behandlung. Bei der Besichtígung erweist sich die linke Gesichtshälfte als bla $B$, leicht cyanotisch; sie fühlt sich kalt an; die rechte ist von normaler Farbe und Temperatur. Das linke Auge ist offen und sein Augapfel geht beim Versuch dasselbe zu schließen nach oben. Die linke Augenbraue steht niedriger als die rechte, die linke Stirnhälfte ist vollkommen glatt, zeigt keine Falten und ist völlig unbeweglich. Beim Öffnen des Mundes geht die Spalte desselben auf die rechte Seite über; die linke Wange ist unbeweglich; beim Aufblassen des Mundes wird sie wie ein Lappen aufgeblasen. Den linken Mundwinkel kann die Patientin weder heben noch herabsinken lassen. Fehlen der Reaktion auf den galvanischen und faradischen Strom. 
Nach 4 Monate hindurch fortgesetzter jedesmal 20 Minuten währender Applikation eines Dampfstrahls von $1 / 20 \mathrm{Atm}$. Druck auf die linke Gesichtshälfte mit Massage des Gesichts glich sich die Gesichtsfarbe beider Wangen aus. Auch die Temperatur der linken Wange zeigte keinerlei Abweichung von der Norm. Die Patientin vermochte frei die linke Augenbraue in die Höhe zu ziehen, das linke Auge $\mathrm{zu}$ schließen und den linken Mundwinkel emporzuziehen. Beim Öffnen des Mundes ist infolge des Auftretens einer Kontraktur in der linken Wange eine Asymmetrie in anderer Richtung zu bemerken. Jetzt reagiert der obere Ast des VII. Cerebralnerven bei $7 \mathrm{~cm}$, der untere bei $3 \mathrm{~cm}$ auf den faradischen Strom.

Die übrigen analogen Fälle von Behandlung veralteter Neuritisfälle mit heißen Prozeduren führe ich hier, um Wiederholungen zu vermeiden, nicht an.

\section{II.}

Eine viel bessere und außerdem schnellere Wirkung übt diese Behandlung in akuten Neuritisfällen, so z. B. bei nach Diphtheritis auftretenden Paralysen. Ich will hier 3 solche Fälle aufführen:

1. Arzt B., 39 Jahr alt, erkrankte im Oktober 1908 an Diphtheritis, weshalb er mit Antidiphtherieserum geimpft wurde. Als die Temperatur gefallen war, zeigten sich die Anzeichen einer Polyneuritis. 3 Monate nach Ablauf der Erkrankung, und zwar den 27. Januar $1909 \mathrm{kam}$ er als ambulatorischer Patient in meine Bebandlung. Er klagte über allgemeine Schwäche, Gefühl von Ameisenkriechen in den Fingern, Herzklopfen, Abmagerung.

Mäßige beiderseitige Atrophie der MM. interossei, der Thenaren und Hypothenaren, sowie der Wadenmuskulatur. Die grobe Muskelkraft mehr in den Händen als in den Füßen rermindert. Die Sensibilität ist in den distalen Teilen der Extremitäten, und zwar in den Händen mehr als in den Füßen herabgesetzt. Die Sehnenreflexe von den MLI. supinator longus und triceps auf beiden Seiten erhalten; Knie- und Achillessehnenreflex gar nicht vorhanden. Pupillen normal; alle Arten von Pupillenreaktionen normal. Die distalen Teile der Extremitäten sind kalt. Puls 120. Keine Herzgeräusche. Puls in den AA. dorsales pedis und tibiales anticae sehr schwach.

Die Behandlung bestand in den obenerwähnten Hyperämie erzeugenden Prozeduren und wurde 2 Monate lang fortgesetzt. Der Erfolg war folgender: Zunahme des Körpergewichts um $5 \mathrm{~kg}$, Erhöhung der motorischen Sphäre, Verschwinden der Schmerzen und Parästhesien und Wiederauftreten der Sehnenreflexe. Puls 80 , in den AA. dorsales pedis war er normal geworden. Bereits nach der ersten Woche der Behandlung stellte sich an den FüBen die normale Hautfarbe ein; sie fühlten sich warm an, und ihr Puls war, wie bereits erwähnt, voll und leicht palpabel geworden.

2. O.S.K., $29 \mathrm{Jahr}$ alt, überstand im September 1909 eine Diphtherie, nach der sich bei ihr Herzklopfen, Schluckbesehwerden, Doppeltsehen, Unmöglichkeit zu lesen und aus der Nähe zu sehen einstellten. Außerdem traten nach diesen akuten Erscheinungen, die sich, das Herzklopfen ausgenommen, bald besserten, Schmerzen in sämtlichen Extremitäten, Ödeme derselben, Sensibilitätsverlust, Gehstörungen und ein derartiger Verfall der groben Muskelkraft in allen willkürlichen Muskeln auf, daß die Kranke fast gar keine Treppen steigen konnte und nur mit Mühe durch die Zimmer ging.

Bei der Besichtigung der Kranken am 4. Februar 1910 wurde folgendes gefunden: Beträchtliche Abmagerung, cyanotische Verfärbung der Extremitäten, insbesondere der Füße, die sich kalt anfühlen; Ödem der letzteren. Puls 
in den AA. dorsales pedis, tibiales anticae, radiales und ulnares sehr schwach, nur mit Mühe palpabel. Herabsetzung des Muskeltonus, namentlich der Unterund Oberschenkelsegmente. Scharf ausgeprägte Muskelatrophie des äußeren Teiles beider Unterschenkel, der Unterarme, der Thenaren und Hypothenaren, sowie der MM. interossei. Willkürliche Extension der Zehen und Füße nicht möglich. Beträchtliche Herabsetzung der groben Muskelkraft in den Händen und Oberschenkeln. Gang scharf ausgeprägt paretisch-ataktisch. Hautreflexe von der Fußsohle und sämtliche Bauchreflexe fehlen. Elektrische Reaktion der NN. peronei, tibiales, crurales, mediani und ulnares auf den galvanischen (bis auf 9-11 M.A.) und faradischen Strom hochgradig (bis auf 3,5 cm) herabgesetzt.

Die Behandlung bestand in denselben Hyperämie erzeugenden Prozeduren (und zwar in lokaler hydraulischer Massage, Dampfspray, Heißluftkasten) mit Sinusoidalbädern und heißen Duschen zum Schluß. Nach Monatsfrist seit dem Beginn der Behandlung begann die Kranke sicher zu gehen und frei Treppen zu steigen; die Schmerzen verschwanden; Sensibilität, sowie Sehnen- und Hautreflexe hatten sich wiedereingestellt; es war aber noch eine gewisse Muskelatrophie an den Händen erhalten geblieben, und die elektrische Reaktion blieb wie früher herabgesetzt; die Hautfarbe war normal, die Ödeme waren verschwunden; der Puls in den peripheren Arterien normal; bereits nach 6 Séancen war die cyanotische Verfärbung der Füße nicht mehr vorhanden, und die Haut derselben fühlte sich nicht. mehr kalt an. Der Puls in den AA. dorsales pedis und tibiales anticae stellte sich in der Mitte der 2. Behandlungswoche wieder ein.

3. E. I. O., 36 Jahr alt, 5 Jahr verheiratet. Machte Anfang September 1909 eine Diphtheritis durch. Hiernach entwickelten sich Parese der NN. oculomotorii, Parese der Akkommodation, Schluckstörungen, hochgradiges Sinken der groben Muskelkraft, insbesondere in den Beinen: die Patinetin konnte. sogar auf ebener Diele nur mit Mühe gehen. Die Erscheinungen seitens der Augen und die Schluckbeschwerden verschwanden bald. Was nun die übrigen Symptome anbelangt, so hatten sich dieselben während der letzten Zeit noch mehr verstärkt, und zwar gesellte sich zu der Schwäche der Beine einerseits Abmagerung, anderseits - Ödem der Füße, Kältegefühl in denselben und Cyanose. Ganz besonders wurde die Kranke durch folgende Beschwerden beunruhigt:- beständiges Schwänken und Unsicherheit beim Gehen, ein Gefühl ron Vertaubtsein in Händen und Füßen und von Brennen in allen Fingern und Zehen, Tag und Nacht andauernde quälende Schmerzen in sämtlichen Extremitäten, Herzklopfen und Atemnot.

Bei der am 10. März 1910 vorgenommenen objektiven Untersuchung wurde gefunden: scharf ausgeprïgter paretisch-ataktischer Gang: die Kranke schleppte die Beine auf dem Boden nach, wobei sie stark nach beiden Seiten hin schwankte; die Füße sind ödematös geschwollen, cyanotisch, fühlen sich kalt an. Der Puls in den AA. dorsalis pedis und tibiales nur mit Mühe fühlbar. Abmagerung des Unterhautzellgewebes, atrophische Erscheinungen in der Muskulatur des Thenar und Hypothenar, der Unterarme, beider Oberschenkel und der äußeren Teile beider Unterschenkel. Kaum merkliche willkürliche Bewegungen der Zehen; ebenso sind auch Flexion und Extension in den Fußgelenken abgeschwächt. Die grobe Kraft der MM. quadric. ist stark herabgesetzt. Taktile Sensibilität, Temperatur, Muskel- und Schmerzgefühl, insbesondere in den Füßen, Unterschenkeln, Händen und Unterarmen herabgesetzt. - Hautreflexe von beiden Füßen, Sehnenreflexe (von der Achillessehne), Kniereflexe, Reflexe von den MM. supinatores longi und von den MM. tricip. fehlen. Pupillen normal. Puls 130-140. Herz um 1 Finger breit nach links über die Mammillarlinie hinaus erweitert; Geräusche an allen Klappen. Leber vergrößert, empfindlich auf Druck. Galvanische Reaktion der NN. peronei, tibiales, crurales und ulnares unter der Norm, so daß man einen 
galvanischen Strom von 7-5 MI.-A. mehr nehmen muß als in der Norm. Die faradische Erregbarkeit ist ebenfalls herabgesetzt, weshalb man die Rollen um $2-\overline{c m}$ näher aneinanderrücken muß als in der Norm, um eine Erregung der NN. peronei, tibiales, ulnares und mediani zu erhalten.

Verordnet wurden die obenerwähnten Hyperämie erzeugenden Prozeduren. Nach Verlauf eines Monats seit dem Beginn der Behandlung begann die Kranke ohne jede Spur von Ataxie zu gehen, und Treppen vermochte sie frei hinauf, und hinab zu steigen. Die Ödeme, die Cyanose und das Kaltwerden der Extremitäten verschwanden bereits nach einwöchentlicher Behandlung. Der Puls in beiden AA. dorsales pedis, sowie in den AA. radiales und ulnares wurde nach $6-8$ Séancen mit Anwendung der hydraulischen Massage normal; mit einem ebenso vollen Pulse machte die Patientin die ganze weitere Kur durch. Die Schmerzen hörten auf; die Sehnenreflexe stellten sich wieder ein. Die Herzdämpfung verringerte sich: die linke äußere Herzgrenze befindet sich jetzt einen Finger breit nach innen von der Mammillarlinie. Beim Auskultieren keine Geräusche mehr.

III.

In den oben aufgeführten kurzen Krankengeschichten lenken mehrere Umstände ganz besonders die Aufmerksamkeit auf sich: 1. Ist aus den objektiven Befunden und den subjektiven Klagen der Patienten ersichtlich, da $\beta$ in allen Fällen die Anzeichen einer gestörten peripheren Blutzirkulation in den paralysierten Körperteilen vorhanden waren, und zwar kleiner, kaum merklicher Puls, eine sich kalt anfühlende Extremität, Cyanose derselben, in einigen Fällen Ödeme usw.; 2. kamen die ersten Resultate der angewandten Behandlung in einer Besserung des peripheren Blutumlaufs zum Ausdruck: der Puls wurde besser, die Kranken hatten kein Kältegefühl mehr in den Extremitäten, die Haut wurde warm, die cyanotische Verfärbung derselben verschwand. Alles das sind Anzeichen davon, daß sich die peripheren Gefäße erweitert haben, und die Blutzufuhr nach den von der Paralyse betroffenen Körperteilen sich verstärkt hat. Folglich hat sich unter dem Einfluß der verordneten Maßnahmen eine dauernde Hyperämie eingestellt. 3. Die scharf ausgeprägte Besserung der Herzsymptome. 4. Die mehr oder weniger vorgeschrittene Wiederherstellung der Funktionen der peripheren Nervenstämme.

Was die unter 3. erwähnten Erscheinungen anbelangt, die darin zum Ausdruck gelangten, daß das bis dahin erweiterte und beim Auskultieren Geräusche aufweisende Herz auf seinen normalen Umfang zurückging, wobei die in demselben zur Beobachtung gelangenden Geräusche während der Anwendung lokale Hyperämie hervorrufender Mittel verschwanden, so werde ich an anderer Stelle ausführlich hierïber handeln (Neurol. Centralbl., Uber die Wirkung der hydraulischen Massage. 1914), hier will ich nur diese überaus interessante Tatsache als solche vermerken.

Was nun die unter 4. erwähnte Erscheinung, und zwar das Eintreten der schnellen Besserung im Bereich des peripheren Nervensystems be- 
trifft - so hat man, um hierüber ins klare zu kommen, die Fähigkeit des erkrankten Nerven zur Regeneration in Erwägung zu ziehen und au ßerdem die gegenseitige Einwirkung der peripheren Gefäße und Nerven aufeinander zu berücksichtigen.

Die Frage von der Regenerationsfähigkeit der peripheren Nervenstämme ist gegenwärtig durch zahlreiche experimentelle Untersuchungen vollkommen aufgeklärt, und sämtliche Autoren sind zu dem Sehlusse gelangt, daß diese Fähigkeit des Nerven ungeheuer groß ist ( $\mathrm{B}$ ü ng ner ${ }^{6}$ ), Howel und $\mathrm{Huber}{ }^{13}$ ), Bethe ${ }^{4}$ ), Lapinsk $y^{21}$ b) u. a.). Wenn man, z. B. bei einem Hunde unter Schonung der großen Gefäße der Extremitäten den ganzen Plexus clavicularis (Lapinsky ${ }^{21}$ b) oder die 3 Beinnerven - NN. cruralis, obturatorius und ischiadicus durchtrennt, wonach die Extremität völlig paralysiert und gefühllos wird, so werden die durchtrennten Nerven ungeachtet dessen, da $B$ das Tier unter dem EinfluB dieser schmerzhaften Operation stark an Körpergewicht abnimmt, und obwohl es keinerlei besondere Pflege erfährt und weder Bäder, noch Massage, noch Elektrisation angewandt werden - stets wiederhergestellt. Die mikroskopische Untersuchung zeigt hierbei, daß die in peripheren Abschnitt vollkommen zerfallene Nervenfaser sich wieder regeneriert und anfänglich einen kahlen aus einzelnen Gliedern bestehenden Achsenzylinder vorstellt, die sich später zu einem rosenkranzförmigen Faden zusammenschließen und schließlich eine Myelinscheide erhalten. Die regenerierte Faser des peripheren Abschnitts verlängert sich und trifft, indem sie aus der Schnittfläche hervortritt, auf eine ebensolche aus dem Querschnitt des zentralen Abschnitts hervorwachsende Faser, verschmilzt mit ihr, und auf diese Weise wird eine kontinuierliche Faser erhalten. In allen diesen Fällen erwiesen sich die großen Gefäßstämme gleichfalls als verletzt, die Blutzirkulation in der betroffenen Extremität blieb jedoch mehr oder weniger zufriedenstellend. In einigen Fällen diente sogar nicht einmal der Umstand als Hindernis für die Regeneration des Nerven, daß sich in der Operationswunde ein eiteriger Prozeß herausbildete. Die Regeneration des Nerven, und zwar seines peripheren Abschnitts ging desto schneller vonstatten, je besser und näher die Schnittflächen seines zentralen und peripheren Abschnitts aneinanderlagen, wobei der Nerv sich im Laufe von 3 Monaten so gut regenerierte und verwuchs, daß es mitunter sogar schwer war, die Narbe an der Stelle der früheren vollkommenen Durchtrennung desselben aufzufinden.

Die Wiederherstellung des Nerven, und zwar gerade seines peripheren Abschnitts erfolgte auch in dem Falle, wenn derselbe gleichzeitig an 2 Stellen durchschnitten und der exzidierte Teil völlig entfernt worden war, oder sogar dann, wenn beide Schnittenden in der Weise von der Umgebung abgelöst und nach verschiedenen Richtungen umgebogen wurden $\left(B\right.$ ethe $\left.{ }^{4}\right)$, Lapinsky $\left.{ }^{21}\right)$ ), daB die sich regenerierenden Fasern des zentralen und peripheren Abschnitts nicht zusammentreffen konnten. Der durchtrennte oder unterbundene Nerv gelangte nur unter einer Bedingung nicht zur Regeneration, und zwar bei unzureichender Blutzufuhr, z. B. bei vollkommener Unterbindung eines großen nährenden Gefäßstammes; unter solchen Verhältnissen traten jedoch bereits die Erscheinungen der Gangrän der Extremität auf.

Auf Grund von zahlreichen nach dieser Richtung angestellten Beobachtungen konnte man sich davon überzeugen, daß sich der Nerv unter jederlei ungünstigen Verhältnissen regeneriert, wenn ihm nur gleichzeitig eine ausreichende Blutmenge zugefïhrt wird. Man ist daher gewissermaßen zu der Annahme berechtigt, daß als die einzige die Regene- 
ration des Nerven günstig beeinflussende Bedingung das Erhaltensein der Blutzirkulation in der betreffenden Extremität erscheint.

Wenn man die experimentellen Befunde in Betracht zieht, so kann man behaupten, daß die Regeneration des Nerven von den sonstigen, noch wenig bekannten Verhältnissen abgesehen, ohne Erhaltensein einer normalen Blutzirkulation nicht möglich ist. Die Bedeutung dieser letzteren für die Regeneration des Nerven wird sich in noch höherem Grade bei der kritischen Bewertung der gegenseitigen Beziehungen überhaupt und der Einflüsse a) der normalen peripheren Nerven auf den Zustand der in ihrem Bereiche gelegenen Gefäße und umgekehrt, b) der Gefäße auf die Lebenstätigkeit der von ihnen versorgten Nerven im Besonderen herausstellen.

Um den Einfluß aufzuklären, den eine zeitweilige Unterbrechung des Blutumlaufs auf den gesunden Nerven haben kann, wurde die Unterbindung [Leser,${ }^{24}$ ) La pins $\mathrm{k} \mathrm{y}^{21 \mathrm{c}}$ )] der Art. femor. bei Kaninchen vorgenommen, wobei man sich davon überzeugen konnte, daß die zeitweilige Unterbrechung der Blutzirkulation den Zerfall der Myelinscheide und andere die parenchymatöse Neuritis charakterisierende Erscheinungen nach sich zieht; wenn aber die Blutzufuhr schnell wiederhergestellt wird, bleibt die Zerstörung nur auf die oberflächliche Schicht der Myelinscheide beschränkt. Bleibt aber die Blutzirkulation im Verlaufe einer sehr langen Zeit erschwert, so treten außerdem noch Wucherungen der Scheidenhüllen des Nervenstamms auf (Nikolski-Lawren. tjew ${ }^{21}$ ), Dutil - Lam $y^{25}$ ), Muravieff ${ }^{27}$ ), Lapinsk $y^{21 c}$ )]. In den beiden erstgenannten Fällen tritt das Bild der Paralyse infolge von Neuritis hervor; im letzterwähnten verläuft die Erkrankung günstiger, ist aber durch äußerst starke Schmerzen charakterisiert, wobei motorische Sphäre, Sensibilität und Reflexe längere Zeit hindurch mehr oder weniger normal bleiben können.

Anderseits ist es dank einer ganzen Reihe von klinischen und experimentellen Arbeiten gelungen, festzustellen, daß jede Paralyse der peripheren Nerven eine Veränderung in der Blutzirkulation und in den Blutgefäßen selbst nach sich zieht, und zwar gerade in der Weise, daß die erstere sich verändert, bevor anatomische Veränderungen in den Gefäßen auftreten. Bei jeder akuten Entwicklung einer Affektion der peripheren Nerven erfolgt eine kurzdauernde Beschleunigung des Blutumlaufs, die sodann von einer dauernden Verlangsamung desselben abgelöst wird (Lapinsk $\mathrm{y}^{21 a}$ ).

Was nun die Gefäße anbelangt, so ist auch in ihnen ein genau bestimmtes Spiel und ein Wechsel der Erscheinungen zu vermerken, und zwar folgen auf eine kurzdauernde Erweiterung derselben, d. $h$. sowohl der Arterien als auch der Venen, Verdickung ihrer Wände, Intimawucherung, Verengerung des Lumens an den einen Stellen und 
variköse Erweiterung desselben an anderen [Fraen kel ${ }^{15}$ ), Giovann $\mathbf{i}^{14}$ ) Lewasche ${ }^{20}$ ), Lapinsk $y^{23}$ ) u.a.].

Als eine Folge der Erkrankung jedes Nerven tritt daher eine Störung der peripheren Blutzirkulation auf. Da aber als unerläßliche hauptsächliche Bedingung für die Regeneration des Nerven gerade das Erhaltensein des Blutumlaufs in der paralysierten Extremität erscheint, da ferner zur Aufrechterhaltung des normalen Zustandes (sowohl des intakten als auch des sich regenerierenden Nerven) eine normale Blutzirkulation im paralysierten Körperteil erforderlich ist, so hat man es bei der Behandlung der peripheren Paralysen mit 2 Erscheinungen zu tun, die voneinander abhängig sind und der Wiederherstellung des paralysierten Körperteils Hindermisse in den Weg legen: die Paralyse des Nerven verursacht Störungen der Blutzirkulation, verlangsamt dieselbe, leistet der Degeneration der Gefäßwand und der qualitativen und quantitativen Veränderung des Nahrungsplasmas Vorschub usw.; anderseits muß man, damit die Regeneration des Nerven erfolgen soll, zuerst die Blutzirkulation verbessern, die sich nicht in allen Fällen spontan wiederherstellen kann, da sich mitunter anatomische Veränderungen in den Gefäßen hindernd in den Weg stellen. Hieraus folgt klar, daß man zur Regeneration des Nerven und zur Wiederherstellung seiner Funktionen, die lokale Blutzirkulation künstlich heben und Maßnahmen zur Erweiterung der Gefäße in Anwendung bringen muß, in deren Verbreitungsgebiet der erkrankte Nerv verläuft. Man hat folglich eine Hyperämie im Bereich des betroffenen Nerven zu erzeugen, dessen ernährende Gefäße, ebenso wie die des Bereichs, in dem dieser Nerv verläuft, ihre normale Lebenstätigkeit eingebüßt haben.

Die Bedeutung der arteriellen Hyperämie für die Behandlung verschiedener Prozesse wird empirisch z. B. durch die Wirkung von heißen Umschlägen, Saug- und Schröpfapparaten, Kompressen, Blutentziehungen sowie durch den Effekt der Laparotomie, bei Tuberkulose des Peritoneums, der Anwendung von ableitenden Prozeduren, der sog. Rubefacientia, von Excitantia exteriora usw. bestimmt.

Beobachtungen am Krankenbett haben gezeigt, daß die Hyperämie in verschiedener Weise auf die kranken Gewebe einwirkt. Einige von den entzündlichen Krankheitsprodukten werden dank der Hyperämie resorbiert, verschiedene abgestorbene Teile lösen sich hierbei ab, Wunden granulieren gut, Exsudate werden resorbiert. Das weist auf die Erhöhung der Lebensprozesse der Zelle dank der erhöhten Blutzufuhr hin.

Experimentell ist auch die bakterizide Wirkung der Hyperämie festgestellt worden.

Es ist häufig der Versuch gemacht worden, im Laboratorium auf dem Wege des Tierexperiments die Bedeutung der Hyperämie als eines die Regeneration der Gewebe begünstigenden Agens klarzustellen. 
Besonders reich an derartigen Versuchen ist die Epoche der Entdeckung der Vasomotoren, als dem Experimentator die Möglichkeit gegeben war, ganz nach Belieben in dem einen oder anderen Bereich eine erhöhte Blutzufuhr zu erzeugen.

Ogle $\left.{ }^{30}\right)$, Schiff $\left.\left.{ }^{34}\right),{ }^{35}\right)$, Pye-Smith ${ }^{31}$ ), Stirling ${ }^{36}$ ), Zechanows $\mathrm{ky}^{42}$ ) haben durch Durchtrennung des N. sympathicus beim Kaninchen Hyperämie des Ohres hervorgerufen und sind zu der tberzeugung gelangt, daß diese Operation zu einer Verstärkung des Wachstums des Ohres und seiner Haare führt.

Kassowitz ${ }^{17}$ ), Schiff ${ }^{34}$ ), ${ }^{35}$ ), $\mathrm{Kusmin}{ }^{18}$ ) haben die Bedingungen der Callusbildung beim Heilen des Knochens nach der Durchtrennung des Nerven studiert und sind zu der Utberzeugung gelangt, daß die hierbei auftretende Hyperämie der Regeneration des Knochens Vorschub leistet.

Broca ${ }^{3}$ ) sah verstärktes Wachstum der Nägel nach Verletzung des N. radialis. Snelle $\mathbf{n}^{37}$ ) fand auf der Seite des durchtrennten Cervicalsympathicus eine beschleunigte Aufsaugung eines Exsudats und beobachtete, daß eine Hautwunde am Ohr auf der normalen Seite in 14 Tagen heilte, während auf der operierten Seite dazu nur 10 Tage erforderlich waren. Ebenfalls in seinen Versuchen lief eine Entzündung der Hormhaut nach Beizen derselben mit Essigsäure auf der Seite des durchschnittenen N. sympathicus schneller ab. Die Ursache hierfür sieht Snellen in der Erhöhung der osmotischen Prozesse zwischen der Blutnährflüssigkeit in den erweiterten Gefäßen der paralysierten Seite und der Gewebsflüssigkeit.

Weber ${ }^{41}$ ) legte nach der Exstirpation des oberen Halsganglions des N. sympathicus auf der einen Seite eine Hautwunde auf der operierten Seite an und vermerkte einen lebhafteren und schnelleren Heilungsprozeß gerade auf der Seite der Hautwunde.

Sinitzin ${ }^{38}$ ) hat eine erhöhte Widerstandsfähigkeit der Hornhaut verschiedenen Reizmitteln gegenüber auf der Seite des durchtrennten N. sympathicus vermerkt, woraus man auf eine größere Regenerationsfähigkeit der der Hyperämie unterworfenen Gewebe schließen darf.

Danilews $\mathrm{k}^{8}$ ) excidierte nach Durchschneidung des Halssympathicus bei Kaninchen Stückchen vom Ohr und erhielt auf der operierten Seite einen lebhafteren und intensiveren Entzündungsprozeß, die Blutextravasate gelangten schneller zur Resorption, und die Gewebsdefekte heilten ohne sichtbaren Verlust. Hieraus folgert Danilewsky, daB die Hyperämie günstig auf die Regeneration des Gewebes einwirkt.

Lei $\mathrm{k}^{\mathbf{2 2}}$ ) excidierte nach Durchtrennung des $\mathrm{N}$. sympathicus oder nach Exstirpation des oberen Halsganglions bei Kaninchen aus beiden Ohren je einen Gewebssektor und beobachtete hierauf im Verlaufe vieler Tage den Heilungsprozeß des Defektes auf beiden Seiten, wobei sich erwies, 
daß die Heilung auf der operierten Seite schneller von statten ging und der Gewebsdefekt sich auf der operierten Seite um 6 und sogar um volle 12 Tage schneller schloß als auf der Seite mit dem intakten N. sympathicus. Bei Kontrollversuchen, während welcher die N. sympathici beiderseits intakt blieben, fand Leik keinerlei Unterschied in der Heilungszeit des linken und rechten Ohres. Den Umstand, daß sich die Wirkung der Durchtrennung des Sympathicus in stärkerem Wachstum auf der operierten Seite äußert, erklärt L e i k durch die arterielle Hyperämie auf der Seite mit dem durchtrennten Sympathicus.

In einer anderen Versuchsreihe brachte Leik an beiden Ohren je einen tiefen penetrierenden Defekt bei und fand nach gleichzeitig auf der einen Seite ausgeführter Durchtrennung des N. sympathicus oder Exstirpation eines Ganglion supremum nach einiger Zeit neben diesen penetrierenden Wunden je einen großen Wulst von neugebildeten Elementen; der auf der operierten Seite gelegene Wulst war höher, und hier war gleichzeitig auch eine stärkere Hyperämie zu bemerken. In diesen Versuchen ging die Heilung des ganzen Defektes am Ohr auf der operierten Seite (wo die stärkere Hyperämie bestand) schneller von statten, und nur in 2 Fällen erfolgte die Heilung in gleichem Tempo und erreichte zu derselben Zeit ihren Abschluß.

In dieser Versuchsreihe waren die Beobachtungen nicht genügend rein, und das vielleicht aus dem Grunde, weil mit der Beseitigung der Regulierung von seiten des vasomotorischen Nervensystems nach der Durchtrennung ${ }^{\prime}$ es Nerven die erhaltene Hyperämie nicht im erforderlichen Maße von den Geweben ausgenutzt werden konnte, denen bei dieser Versuchsanordnung der regulierende Einfluß der Nervenzentren entzogen war. Von ungeheurem Interesse ist daher eine andere Versuchsanordnung, bei der das vasomotorische System intakt bleibt und die Forscher die Hyperämie mit Hilfe von erhöhter Temperatur aufweisenden Apparaten hervorrufen. In dieser zweiten Versuchsreihe erweist sich die Einwirkung der Hyperämie als durch die Anwendung von andauernder Wärme verstärkt.

Penzo ${ }^{32}$ ) hat Versuche an Kaninchen vorgenommen, denen er die Ohrlöffel oder Pfoten dauernd erhitzte oder abkühlte. In einem speziell zu diesem $Z$ wecke von ihm konstruierten Apparat hielt er den einen Ohrlöffel des Kaninchens bei $38^{\circ} \mathrm{C}$, den anderen aber bei $10^{\circ} \mathrm{C}$ und konnte sich davon überzeugen, daß sich der erstere im Zustande der Hyperämie, der letztere dagegen in dem der Ischämie befand. Der erstere begann am 2. Versuchstage sein Integument zu verlieren und zu schelfern. Nach dem Einstellen des Versuches behielt dieses $\mathrm{Ohr}$ seine erhöhte Temperatur noch im Laufe von 20-25 Minuten; gleichzeitig blieb das andere Ohr kalt und blutarm. Doch nach Verlauf von 30 Minuten glichen sich die Temperaturen beider Ohren aus, und nach 60 Minuten 
seit dem Beginn des Versuches wurde das hyperämierte kalt und anämisch, das abgekühlte warm und hyperämisch.

Der Autor stellte feine Schnitte von beiden Obren her und gelangte, nachdem er auf ihnen die mitotischen Figuren im Epithel des Ohrlöffels aufgesucht, zu der Uberzeugung, daß die Zahl der Mitosen im erhitzten Ohrlöffel viel größer war als im abgekühlten.

In einer anderen Serie von Versuchen hielt Penzo die Ohrlöffel der als Versuchstiere dienenden jungen Kaninchen wäbrend vieler Wochen, den einen in einer Temperatur von $38^{\circ} \mathrm{C}$, den anderen in einer solchen von $10^{\circ} \mathrm{C}$, wobei er vermerkte, daß das Wachstum der Ohrlöffel der Kaninchen ungleichmäßig von statten ging, und zwar wuchs der erhitzte Ohrlöffel viel schneller als der der Abkühlung ausgesetzte.

In einer dritten Versuchsreihe nahm Penzo die Heilung von Knochen- und Knorpelbrüchen sowie von Hautschnittwunden bei Temperaturen von $38^{\circ} \mathrm{C}$ und $10^{\circ} \mathrm{C}$ vor, wobei sich herausstellte, da $B$ die Knorpel in der Wärme ohne Narbe verheilten, während es in den abgekühlten zu einer Nekrose kam.

Aus dem oben Gesagten ist ersichtlich, daß die Experimente, mit deren Hilfe die Bedeutung der Hyperämie klargestellt werden sollte, mit unzweifelhafter Klarheit die ganze Wichtigkeit dieses Prozesses für den Regenerationsvorgang einiger tierischer Gewebe dargetan haben. Auf Grund meiner hier dargelegten klinischen Beobachtungen dürfte man zu der Annahme berechtigt sein, daß die Hy perämie a uch a uf die Regeneration des peripheren Nervensystems günstig einwirkt, und zwar nicht nur in frischen, sondern auch in vernachlässigten, inveterierten und chronischen Fällen.

Wenn wir uns nun wieder unseren Fällen und denjenigen Prozeduren zuwenden, die den belebenden Effekt der Hebung der lokalen Blutzirkulation in stärkstem Maße hervorriefen, so will mir scheinen, daß als die in dieser Hinsicht am meisten belebende Prozedur die hydraulische Massage zu gelten hat, die (wovon noch besonders gehandelt werden soll) eine Gefäßerweiterung nicht nur in der Haut, sondern auch in den Weichteilen der Extremität, in ihren Muskelgefäßen und in den Blutbahnen der Gefäßnervenbündel hervorruft. Die beiden letzterwähnten Arten von Blutbahnen, die sich der Einwirkung anderer Heilmethoden gegenüber als sehr wenig zugänglich erweisen, sind aber gerade für die Regeneration und regelrechte Funktion der Nervenfasern ganz besonders wichtig. Daher scheint es mir, daß man den wesentlichsten Nutzen im Sinne der Regeneration der peripheren Nervenstämme gerade von der hydraulischen Massage zu erwarten hat, die eine Hyperämie der tiefen Gefäßnetze der paralysierten Extremität erzeugt. Was nun den Dampf, das Licht, die heiße Luft und die Sinusoidalstrombäder anbelangt, so kommt diesen Prozeduren wahrscheinlich eine nur nebensächliche 
Bedeutung zu, da ihre Einwirkung auf die oberflächlichen Körperteile, insbesondere auf die Haut, beschränkt ist. Die bei diesen Prozeduren erhaltene oberflächliche Hyperämie pflegte jedoch eine andere Wirkung zu üben, und zwar wurde die Tätigkeit des Drüsenapparats der Haut, insbesondere der Schweißdrüsen, gehoben, so daß sich der Organismus von verschiedenen Toxinen befreien konnte, die die allgemeine Ernährung störten und die Regeneration der Nervenfasern verzögerten. Wenn man auch in chronischen Fällen von Neuritis diesen toxischen Produkten keine besondere Bedeutung beimessen kann, so müssen sie hingegen in akuten Fällen von Polyneuritis eine wesentliche Rolle spielen; als lehrreiches Beispiel hierfür dienen denn auch die oben aufgeführten 3 akuten Polyneuritisfälle mit dem in ihnen erhaltenen vollkommen zufriedenstellenden Ergebnis der angewandten Behandlung.

\section{Literaturverzeichnis.}

1. Angelucci, Alterations trophiques de l'oeil, consécutives à l'extirpation du Ganglion cervical. Arch. italiennes de Biol. 20, 1. 1893.

3. Broca, Gazette des Hôpitaux 42. 1874.

4. Bethe, Allgemeine Anatomie und Physiologie des Nervensystems. 1903.

5. Bruch, Zeitschr. fo wissenschaftl. Zoologie 6.

6. Büng ner, Zieglers Beiträge z. allg. Path. u. pathol. Anat. 10.

7. Beneke, Virchows Archiv. 1872.

8. Da nilewsky, Centralbl. f. Chir. S. 214. 1883.

9. Davidsohn, Über ischämische Lähmung. Erlangen. 1871.

10. Chrostek, Jahrb. f. Psych. 1890.

11. Czihlarz-Helbing, Centralbl. f. allg. Pathol. 1897.

13. Howel - Huber, Journ. of Physiol. 13-14.

14. Giovanni, vgl. Martin. Rev. de Méd. 1875.

15. Fränkel, Wiener klin. Wochenschr. 1896.

16. Joseph, Über den Einfluß der Nerven auf Ernährung... Archiv f. Anat. u. Physiol. 1872.

17. Kassowitz, Centralbl. f. d. med. Wissenseh. 44. 1878.

18. Kusmin, Allgem. Wiener med. Zeitschr. 1882.

19. Kapsammer, a) Wiener klin. Wochenschr. 1897.

b) Langenbecks Archiv 56.

20. Levaschew, a) Centralbl. f. d. med. Wissensch. 1883.

b) Centralbl. f. Nervenheilk. 1880 .

c) Archives slaves de Biol. 1886.

21. La pinsky, a) Arch. Du Bois-Raymond. 1899.

b) Virchows Archiv 183.

c) Deutsche Zeitschr. f. Nervenheilk. 1899, 1900, 1901.

22. Leik, Utber den Einflu B der arteriellen Hyperämie. Archiv f. klin. Chir. 1902.

23. La pinsky, Gefäßdegeneration nach Sympathicusdurchschneidung. Zeitschr. f. Nervenheilk. 1900.

24. Leser, Vollkmanns Sammlung. 1884-1886.

25. Lamy-Dutil, Arch. de la Méd. Expérim. 1893.

27. Muravieff, Medicinskoje Obosrenije. 1895 (russ.).

29. Nikolsky - Lavrentieff, Medicinskoje Obosrenije. 1892 (rass.). 
30. Ogle, Med. Times and Gaz. 1867.

31. Pye-Smith, Journ. of Physiol. 8, 1.

32. Penzo, Über den Einfluß der Temperatur auf die Regeneration der Zellen. Molleschotts Untersuchungen zur Naturlehre. 1894.

33. Samuel, a) Die Regeneration. Virchows Archit 50. 1870.

b) Gewebswachstum bei Störung der Blutzirkulation. Ibidem. 108.

c) Gewebswachstum bei Störung der Innervation 113.

34. Schiff, Compt. rend. de l'Acad. des Sc. 1854.

35. - Schmidts Jahrbïcher $\mathbf{8 9}$.

36. Stirling, Journ. of Anat. and Physiol. 1876.

37. Snellen, v. Donders, Archiv f. holländische Beiträge. 1 Berlin 1858.

38. Sinitzin, Centralbl. f. d. med. Wissensch. S. 161. 1871.

39. Sam uel, Über anämische und neurotische Entzündung. Virchows Archiv 121. 1890.

40. Waller, Compt. rend. des l'Acad. des Sc. 36, 378. 1853.

41. Weber, Verhand. des naturhist. Vereins d. preuß. Rheinlande 21.

42. Zechanovsky, Bedeutung der Durchschneidung des N. sympathicus. Dissertation. St. Petersburg. 1896. 